Hamlington, Peter E.; Krasnov, Dmitry; Boeck, Thomas; Schumacher, Jörg:

Local dissipation scales and energy dissipation-rate moments in channel flow

URN: $\quad$ urn:nbn:de:gbv:ilm1-2014210050

Published OpenAccess: September 2014

Original published in:

Journal of fluid mechanics. - Cambridge [u.a.] : Cambridge Univ. Press (ISSN 1469-7645). - 701 (2012), S. 419-429.

DOI: $\quad$ 10.1017/jfm.2012.170

URL: $\quad$ http://dx.doi.org/10.1017/jfm.2012.170

[Visited: 2014-08-27]

„Im Rahmen der hochschulweiten Open-Access-Strategie für die Zweitveröffentlichung identifiziert durch die Universitätsbibliothek IImenau."

"Within the academic Open Access Strategy identified for deposition by IImenau University Library."

„Dieser Beitrag ist mit Zustimmung des Rechteinhabers aufgrund einer (DFG-geförderten) Allianz- bzw. Nationallizenz frei zugänglich."

"This publication is with permission of the rights owner freely accessible due to an Alliance licence and a national licence (funded by the DFG, German Research Foundation) respectively."

\section{DFG}

Nationallizenzen 


\title{
Local dissipation scales and energy dissipation-rate moments in channel flow
}

\author{
P. E. Hamlington ${ }^{1}$, D. Krasnov ${ }^{2}$, T. Boeck ${ }^{2}$ and J. Schumacher ${ }^{2} \dagger$ \\ ${ }^{1}$ Department of Aerospace Engineering Sciences, University of Colorado, Boulder, \\ CO 80309-0429, USA \\ 2 Institut für Thermo- und Fluiddynamik, Technische Universität Ilmenau, Postfach 100565, \\ D-98684 Ilmenau, Germany
}

(Received 5 December 2011; revised 30 March 2012; accepted 30 March 2012; first published online 10 May 2012)

Local dissipation-scale distributions and high-order statistics of the energy dissipation rate are examined in turbulent channel flow using very high-resolution direct numerical simulations at Reynolds numbers $R e_{\tau}=180,381$ and 590. For sufficiently large $R e_{\tau}$, the dissipation-scale distributions and energy dissipation moments in the channel bulk flow agree with those in homogeneous isotropic turbulence, including only a weak Reynolds-number dependence of both the finest and largest scales. Systematic, but $R e_{\tau}$-independent, variations in the distributions and moments arise as the wall is approached for $y^{+} \lesssim 100$. In the range $100<y^{+}<200$, there are substantial differences in the moments between the lowest and the two larger values of $R e_{\tau}$. This is most likely caused by coherent vortices from the near-wall region, which fill the whole channel for low $R e_{\tau}$.

Key words: turbulent boundary layers, turbulence simulation, turbulence theory

\section{Introduction}

The dissipation of kinetic energy by viscosity in fully developed turbulence occurs primarily at the smallest scales of the flow, where viscous effects overwhelm inertial processes. All such dissipation is assumed to occur near the mean scale $\eta_{K}=\left(v^{3} /\langle\varepsilon\rangle_{x, t}\right)^{1 / 4}$ in the classical picture of turbulence developed by Kolmogorov (1941), where $v$ is the kinematic viscosity and $\varepsilon(\boldsymbol{x}, t)=(v / 2)\left(\partial_{j} u_{i}^{\prime}+\partial_{i} u_{j}^{\prime}\right)^{2}$ is the local energy dissipation rate. The variable $u_{i}^{\prime}$ is the fluctuating velocity and $\langle\cdot\rangle_{x, t}$ denotes an average over all space and time. Prior numerical and experimental studies (see e.g. Sreenivasan \& Antonia 1997; Ishihara, Gotoh \& Kaneda 2009; Wallace \& Vukoslavcević 2010) have shown, however, that $\varepsilon$ is highly intermittent, resulting in local values of $\varepsilon$ that can be orders of magnitude larger than the mean, even for turbulent flows at moderate Reynolds numbers. Such high amplitudes are, by definition, the result of very large velocity gradients, or tiny shear layers across which the velocity varies significantly. The dissipation can thus be connected to a locally varying scale, $\eta(\boldsymbol{x}, t)$, associated with fluctuations in the velocity gradient field. This gives a range of fluctuating scales, $\eta$, over which dissipation occurs, as opposed to the single mean scale $\eta_{K}$. Certain aspects of the resulting distributions of $\eta$ remain unclear, however, including the properties of the smallest scales (corresponding to the largest

$\dagger$ Email address for correspondence: joerg.schumacher@tu-ilmenau.de 
values of $\varepsilon$ ), as well as the universality of the distributions in flows with different large-scale turbulence generation mechanisms. The latter issue is of particular interest in wall-bounded shear flows, where turbulence is created mainly in the anisotropic near-wall region, while the bulk flow away from the walls is expected to be essentially isotropic.

In this work, we address both of these points by using very high-resolution direct numerical simulations (DNS) to study distributions of the local dissipation scale, $\eta$, and high-order statistics of $\varepsilon$ in three-dimensional, fully-developed turbulent channel flow. The properties of $\eta$ have been examined in the past from various perspectives, beginning with studies of turbulence multifractality (Paladin \& Vulpiani 1987; Nelkin 1990; Biferale 2008). Later, Sreenivasan (2004) suggested that the local dissipation scale be written as $\tilde{\eta}(\boldsymbol{x}, t)=\left[v^{3} / \varepsilon(\boldsymbol{x}, t)\right]^{1 / 4}$, allowing fluctuations in the scale to be directly connected to variations in $\varepsilon$. The definition of $\eta$ used in the present study is based on work by Yakhot (2006), which expresses $\eta$ in terms of longitudinal velocity increments, $\delta_{\eta} u_{i}=\left|u_{i}\left(x_{i}+\eta\right)-u_{i}\left(x_{i}\right)\right|$ (summation over repeated indices is not implied), using the relation $\eta\left(\delta_{\eta} u_{i}\right) \approx v$. The connection between this relation for $\eta$ and the Kolmogorov-type scale, $\tilde{\eta}$, is discussed in more detail in the Appendix. The present definition from Yakhot (2006) identifies flow regions - or eddies - of size $\eta$ with local velocity increments corresponding to Reynolds numbers $R e_{\eta}=\left(\delta_{\eta} u_{i}\right) \eta / v \approx 1$. The scale $\eta$ is thus associated with structures where viscous and inertial effects are in approximate balance (Yakhot \& Sreenivasan 2005). Probability density functions (p.d.f.s) of $\eta$, denoted $Q(\eta)$, have been calculated previously using DNS of homogeneous isotropic turbulence (Schumacher 2007) and in experiments on turbulent pipe flow (Bailey et al. 2009) and buoyancy-driven turbulence (Zhou \& Xia 2010). Comparisons of $Q(\eta)$ in isotropic turbulence and on the pipe flow centreline have shown qualitative agreement, indicating that $Q(\eta)$ may have a universal form that is independent of the large-scale turbulence generation mechanism.

Here we extend these prior comparisons by performing the first computational study of $Q(\eta)$ in a wall-bounded turbulent shear flow. We examine $Q(\eta)$ as a function of both the friction Reynolds number, $R e_{\tau}=u_{\tau} L / \nu$, and wall distance, $y^{+}=y u_{\tau} / v$, where $u_{\tau}$ is the friction velocity, $L$ is the channel half-height, and $y$ is the direction normal to the channel walls. Three different Reynolds numbers, $R e_{\tau}=180,381$ and 590, are examined, and we consider wall distances spanning the full extent of the channel, from the viscous sublayer $\left(y^{+}=2\right)$ to the channel centreline. This approach allows us to separate near-wall and bulk-flow regions on the basis of dissipation statistics. We also study the height and Reynolds-number dependence of dissipation moments up to fourth order. The scaling of these moments with the global largescale Reynolds number, $R e=u^{\prime} L / v$, is compared with results from prior studies (Schumacher, Sreenivasan \& Yakhot 2007; Donzis, Yeung \& Sreenivasan 2008) of homogeneous isotropic turbulence, where $u^{\prime}=\left\langle\left(u_{x}^{\prime}\right)^{2}+\left(u_{y}^{\prime}\right)^{2}+\left(u_{z}^{\prime}\right)^{2}\right\rangle_{x, t}^{1 / 2}$. Dissipation moments were examined in Boeck, Krasnov \& Schumacher (2010) and Hamlington et al. (2012) for $R e_{\tau}=180$ and 381, and here we include a third, higher, value of $R e_{\tau}$ in order to test the scaling with $R e$. We will show that the scaling of the moments in the bulk flow near the centreline coincides with the isotropic case if the Reynolds number is sufficiently large.

\section{Direct numerical simulations}

DNS is currently the most feasible approach for studying high-order statistics of $\varepsilon$ in turbulent channel flows, since experimental measurements covering the full channel 


\begin{tabular}{rrrcccccc}
$R e_{\tau}$ & \multicolumn{1}{c}{$R e$} & \multicolumn{1}{c}{$\operatorname{Re}_{U}$} & $N_{x} \times N_{y} \times N_{z}$ & $N_{t}$ & $\Delta x^{+}$ & $\Delta y_{c}^{+}$ & $\Delta z^{+}$ & $\Delta t / t_{c}$ \\
180 & 339 & 2800 & $512 \times 1025 \times 512$ & 403 & 4.4 & 0.55 & 2.2 & 0.1 \\
381 & 712 & 6667 & $1024 \times 1025 \times 512$ & 134 & 2.3 & 1.2 & 2.3 & 0.2 \\
590 & 1160 & 11000 & $2048 \times 2049 \times 1024$ & 81 & 1.8 & 0.91 & 1.8 & 0.2
\end{tabular}

TABLE 1. Simulation parameters: friction Reynolds number $R e_{\tau}$; global Reynolds number $R e=u^{\prime} L / \nu$; flux-based Reynolds number $R e_{U}=U L / v$; grid dimensions $N_{x} \times N_{y} \times N_{z}$; number of temporal snapshots $N_{t}$; streamwise, $\Delta x^{+} \equiv \Delta x u_{\tau} / \nu$, spanwise, $\Delta z^{+} \equiv \Delta z u_{\tau} / \nu$, and centreline wall-normal, $\Delta y_{c}^{+} \equiv \Delta y(y / L=1) u_{\tau} / v$ resolutions; and time step between snapshots, $\Delta t$, normalized by $t_{c}=L / U$. Grid sizes are listed without $2 / 3$ de-aliasing.

height can be complicated by the required small-scale resolution and the need to measure all nine components of $\partial_{j} u_{i}$ (see Wallace \& Vukoslavcević 2010 for a review). Compared to many previous numerical studies at similar or higher values of $R e_{\tau}$ (e.g. Kim, Moin \& Moser 1987; Moser, Kim \& Mansour 1999; Hoyas \& Jimenez 2006), the resolution of the present DNS has been increased by up to a factor of approximately five in each spatial direction in order to accurately measure high-order moments and small-scale statistics; calculation of the fourth-order moment of $\varepsilon$, for example, requires accurate resolution of at least an eighth-order velocity derivative, as discussed in Schumacher et al. (2007). Measuring such high-order derivative moments - which are associated with the finest structures or the steepest gradients in the channel - thus requires resolutions that substantially exceed those used in many prior studies, as shown by Boeck et al. (2010) for $R e_{\tau}=180$. Similarly high resolutions are also used in recent dissipation-rate studies of turbulent channel flow by Abe \& Antonia (2011).

The present simulations are performed using a Fourier-Chebyshev pseudospectral method with $2 / 3$ de-aliasing in all three directions and a second-order time discretization. We solve the Navier-Stokes equations for an incompressible flow given by

$$
\partial_{i} u_{i}=0, \quad \partial_{t} u_{i}+u_{j} \partial_{j} u_{i}=-\partial_{i} p+v \partial_{j}^{2} u_{i},
$$

where $u_{i}$ is the total velocity and $p$ is the kinematic pressure field. Time discretization of the governing equations is carried out using implicit representations for linear terms and the explicit, second-order Adams-Bashforth method for nonlinear terms, resulting in an overall second-order-accurate discretization (Boeck et al. 2010; Hamlington et al. 2012).

The size of the computational domain is $\left(L_{x} \times L_{y} \times L_{z}\right) / L=4 \pi \times 2 \times 2 \pi$ for $\operatorname{Re}_{\tau}=180$ and $2 \pi \times 2 \times \pi$ for $R e_{\tau}=381$ and 590. Temporal snapshots of the velocity and velocity gradient fields are stored every 0.1 convective time units, $t_{c}=L / U$ (where $U$ is the bulk flow velocity at constant flux), for $R e_{\tau}=180$, and every $0.2 t_{c}$ for $R e_{\tau}=381$ and 590. This results in 403, 134 and 81 total snapshots for $R e_{\tau}=180,381$ and 590, respectively. Analysis of the data is carried out in $x-z$ planes parallel to the channel walls and statistical convergence is improved by averaging over all available temporal snapshots, as well as using symmetric planes from the top and bottom halves of the channel. In the following, $\langle\cdot\rangle$ denotes an average over $x-z$ planes, at all times, and at both symmetric planes, and is generally a function of $y$. The fluctuating velocity gradient, $\partial_{j} u_{i}^{\prime}$, necessary to obtain $\varepsilon$ is calculated by subtracting $\left\langle\partial_{j} u_{i}\right\rangle$ at each $y$ from $\partial_{j} u_{i}$ in the corresponding $x-z$ plane. Reynolds numbers and resolution details (both temporal and spatial) for the present simulations are summarized in table 1. 




FIGURE 1. Longitudinal structure functions $S_{2 n}(r) / r^{2 n}=\left\langle\left(\delta_{r} u_{x}\right)^{2 n}\right\rangle / r^{2 n}$, normalized by $\left(u_{\tau} / L\right)^{-2 n}$, at the centreline for $R e_{\tau}=590$. Horizontal dashed lines show analytic-range scaling $S_{2 n}(r) / r^{2 n}=$ const., and vertical dash-dot line shows $r / \eta_{K}=1$.

The adequacy of the resolution used in the present simulations can be examined from the scaling of longitudinal structure functions of the streamwise velocity, denoted $S_{2 n}(r)=\left\langle\left(\delta_{r} u_{x}\right)^{2 n}\right\rangle$. From Taylor expansion of $S_{2 n}(r)$, it follows analytically that $S_{2 n}(r) \sim r^{2 n}$ when $r$ is small. Very fine resolutions are required to recover this analyticrange behaviour, particularly for high Reynolds numbers and large $n$ (Schumacher et al. 2007). Figure 1 shows that, at the centreline for $\operatorname{Re}_{\tau}=590, S_{2 n}(r) / r^{2 n} \approx$ const. for small $r$ up to $n=5$ and for $n=4$ the analytic range is still approximately recovered up to $r \approx 2 \eta_{K}$.

\section{Distributions of local dissipation scales}

Local dissipation-scale p.d.f.s, $Q(\eta)$, are calculated by constructing conditional histograms of the scale $r$ when the local Reynolds number, $\left(\delta_{r} u_{i}\right) r / v$, is near 1 , or more precisely when $0.9 \leqslant\left(\delta_{r} u_{i}\right) r / v \leqslant 1.1$. The p.d.f.s $Q(\eta)$ can then be formally written as $Q(\eta)=P_{r}\left[\eta \mid\left(\delta_{r} u_{i}\right) r / v=1\right]$, where $r \in\left(0, L_{i}\right]$ represents all possible values of the spatial increment, $P_{r}$ is the p.d.f. of $r$, and the conditional set contains all increments, over all points, where $\left(\delta_{r} u_{i}\right) r / v=1$ (Schumacher 2007). Including only values of $r$ satisfying this Reynolds-number criterion isolates flow structures occurring at the cutoff between the inertial and dissipative ranges. Since the analysis is carried out in $x-z$ planes, either streamwise, $\delta_{r} u_{x}=\left|u_{x}(x+r, y, z, t)-u_{x}(x, y, z, t)\right|$, or spanwise, $\delta_{r} u_{z}=\left|u_{z}(x, y, z+r, t)-u_{z}(x, y, z, t)\right|$, longitudinal velocity increments can be used to obtain $Q(\eta)$.

Yakhot (2006) showed that one consequence of the continuum of local dissipation scales is that the crossover scale, denoted $\eta_{2 n}$, between the dissipative and inertial ranges of $S_{2 n}(r)$ depends on $n$ and is given by

$$
\eta_{2 n}=\ell \operatorname{Re}_{\ell}^{1 /\left(\zeta_{2 n}-\zeta_{2 n+1}-1\right)}
$$

where $\ell$ is the turbulent integral scale, we take $R e_{\ell}=u^{\prime} \ell / v$ as the corresponding Reynolds number, and $\zeta_{2 n}$ are the scaling exponents of $S_{2 n}(r)$ in the inertial range. Inserting $\zeta_{2 n}=2 n / 3$ from the Kolmogorov hypothesis (Kolmogorov 1941) into (3.1) 

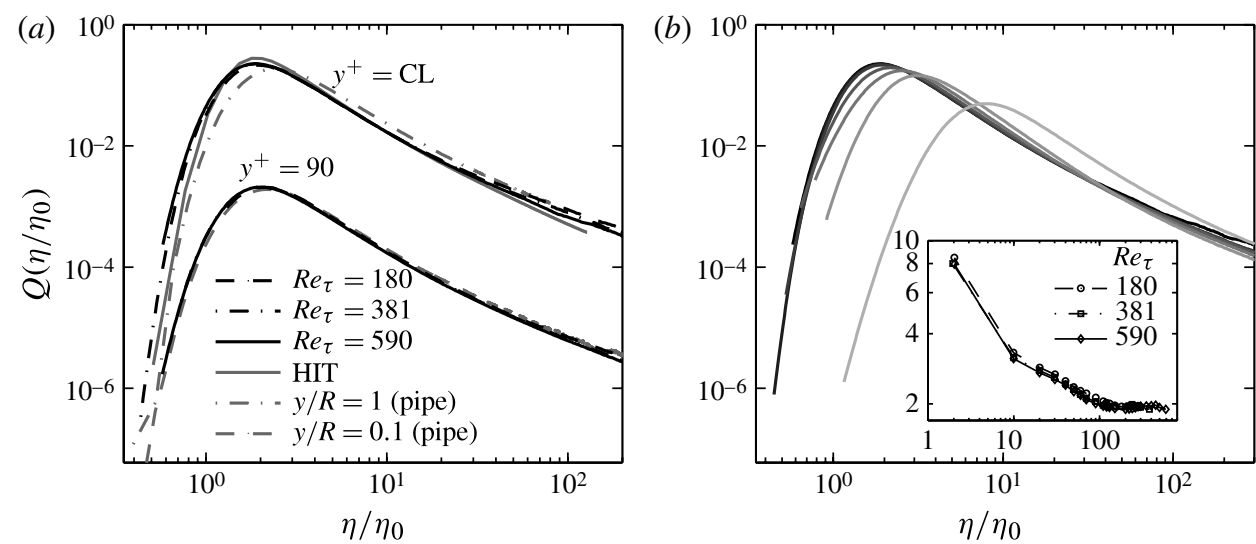

FIGURE 2. (a) Local dissipation scale p.d.f.s $Q\left(\eta / \eta_{0}\right)$ for $R e_{\tau}=180,381$ and 590 at $y^{+}=90$ and the centreline $(\mathrm{CL})$ using streamwise $\left(u_{x}\right)$ velocity increments. Solid lines are cubic splines through the DNS results, and the p.d.f.s for $y^{+}=90$ are shifted down by two decades. Results from DNS of homogeneous isotropic turbulence (HIT) by Schumacher (2007) and turbulent pipe flow experiments by Bailey et al. (2009), with pipe radius $R$, are also shown. (b) Variation of $Q\left(\eta / \eta_{0}\right)$ with $y^{+}$for $R e_{\tau}=590$ using streamwise $\left(u_{x}\right)$ velocity increments. P.d.f.s are shown for $y^{+}=2,10,30,60,120,200$ and 590, corresponding to shades from grey (low $y^{+}$) to black (high $y^{+}$). Solid lines are cubic splines through the DNS data. Inset shows locations of maximum $Q\left(\eta / \eta_{0}\right)$ (vertical axis), denoted $\left(\eta / \eta_{0}\right)_{\max }$, as a function of $y^{+}$ (horizontal axis) for all $R e_{\tau}$.

then gives the classical result $\eta_{2 n}=\ell R e_{\ell}^{-0.75}=\eta_{K}$. The Kolmogorov scale thus appears only if we make explicit use of the classical Kolmogorov theory. A new scale within this framework, $\eta_{0}$, follows by using the well-known intermittency corrections that lead to anomalous scaling exponents (e.g. She \& Lévêque 1994; Sreenivasan \& Antonia 1997). A good approximation for $n<5$ is $\zeta_{2 n}=2 a n-4 b n^{2}$, and using $a=(1+9 b) / 3=0.383$ (which is a consequence of $\left.\zeta_{3}=1\right)$ and $b=0.0166$ then gives

$$
\eta_{0} \simeq \ell \operatorname{Re}_{\ell}^{-1 /(1+a)}=\ell \operatorname{Re}_{\ell}^{-0.72} .
$$

For the channel flow, the $y$-dependence of $\ell$ and $R e_{\ell}$ must also be taken into account, giving $\eta_{0}(y)=\ell(y) \operatorname{Re}_{\ell}(y)^{-0.72}$, where $u^{\prime}(y)=\left\langle\left(u_{x}^{\prime}\right)^{2}+\left(u_{y}^{\prime}\right)^{2}+\left(u_{z}^{\prime}\right)^{2}\right\rangle_{x, z, t}^{1 / 2}$ is used in $\operatorname{Re}_{\ell}(y)$. This $y$-dependent normalization is similar to that used in prior studies (Schumacher 2007; Bailey et al. 2009) and is related to $\eta_{K}(y)$ by $\eta_{0}(y)=\eta_{K}(y)^{1.12}$ $\left[u^{\prime}(y) / \nu\right]^{0.12}$. Values of $\eta_{0}$ are always close to the classical Kolmogorov scale $\eta_{K}$, with $\eta_{0}(y) / \eta_{K}(y)=\operatorname{Re}_{\ell}(y)^{0.03}$.

Figure 2(a) shows $Q\left(\eta / \eta_{0}\right)$ at $y^{+}=90$ and at the centreline using streamwise velocity increments, $\delta_{r} u_{x}$. At both locations, there are only weak variations in $Q\left(\eta / \eta_{0}\right)$ with $R e_{\tau}$; similarly weak Reynolds-number dependence was observed for the pipe flow experiments by Bailey et al. (2009). For large $\eta / \eta_{0}$, all p.d.f.s at both values of $y^{+}$are in close agreement with the pipe-flow measurements and DNS of isotropic turbulence. For small $\eta / \eta_{0}, Q\left(\eta / \eta_{0}\right)$ at the centreline varies only weakly with Reynolds number, and the magnitude and location of the maxima in the p.d.f.s are close to those found in homogeneous isotropic turbulence. There is also good agreement with the pipe-flow results at $y^{+}=90$ for small $\eta / \eta_{0}$, despite the substantially lower Reynolds numbers used in the present simulations. 


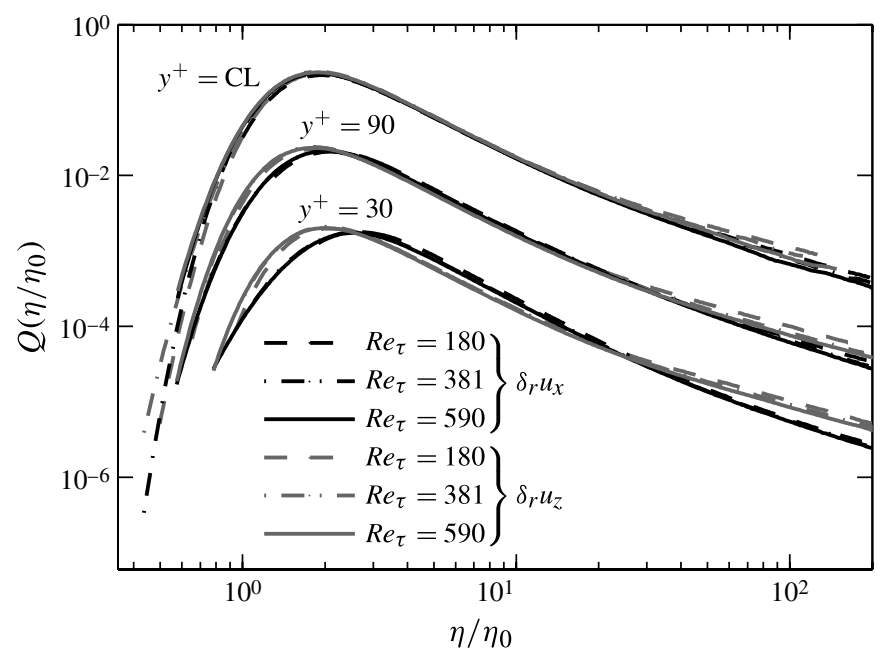

FIGURE 3. Local dissipation-scale p.d.f.s $Q\left(\eta / \eta_{0}\right)$ for $R e_{\tau}=180,381$ and 590 at $y^{+}=30$, 90 , and the centreline $(\mathrm{CL})$ using streamwise $\left(u_{x}\right)$ and spanwise $\left(u_{z}\right)$ velocity increments. Lines are cubic splines through the DNS data, and p.d.f.s for $y^{+}=90$ and $y^{+}=30$ are shifted down by one and two decades, respectively.

While there are relatively weak changes in $Q\left(\eta / \eta_{0}\right)$ with $R e_{\tau}$, the p.d.f.s vary considerably in the near-wall region of the channel. Figure $2(b)$ shows that $Q\left(\eta / \eta_{0}\right)$ is essentially unchanged for $y^{+} \gtrsim 100$, but the location of maximum $Q\left(\eta / \eta_{0}\right)$, denoted $\left(\eta / \eta_{0}\right)_{\max }$, increases as the wall is approached (as seen in the inset). At all $\operatorname{Re}_{\tau},\left(\eta / \eta_{0}\right)_{\max }$ is close to 2 for $y^{+} \gtrsim 100$, but increases for smaller $y^{+}$, with $\left(\eta / \eta_{0}\right)_{\max } \approx 8$ at $y^{+}=2$. Interestingly, the rescaling using viscous wall units results in a near-perfect collapse of the $\left(\eta / \eta_{0}\right)_{\max }$ curves for the two larger values of $R e_{\tau}$, suggesting a robustness of the variations in the p.d.f.s with respect to Reynolds number. The increase in $\left(\eta / \eta_{0}\right)_{\max }$ in figure 2 may be explained by the presence of extended coherent vortices near the wall (Adrian 2007). These vortices increase the velocity correlation length, particularly in the streamwise $(x)$ direction. The condition $\left(\delta_{r} u_{x}\right) r / v \approx 1$ may thus be satisfied for increasingly large $r$, resulting in increased $\left(\eta / \eta_{0}\right)_{\max }$. The fact that this increase occurs over nearly all $y^{+}$in the $R e_{\tau}=180$ case suggests that coherent vortices generated near the wall may dominate the entire channel for small $R e_{\tau}$.

Comparisons of $Q\left(\eta / \eta_{0}\right)$ calculated using streamwise $\left(\delta_{r} u_{x}\right)$ and spanwise $\left(\delta_{r} u_{z}\right)$ velocity increments in figure 3 give similar insights into the channel flow structure. At the centreline and near the wall, there is little difference in $Q\left(\eta / \eta_{0}\right)$ obtained using $\delta_{r} u_{x}$ and $\delta_{r} u_{z}$. As the wall is approached, however, the streamwise p.d.f.s shift to larger $\eta / \eta_{0}$ more rapidly than the spanwise p.d.f.s. This can again be attributed to the presence of extended streamwise vortices near the wall, which result in stronger streamwise velocity correlations and hence smaller differences in $u_{x}$ (as compared to the spanwise differences in $u_{z}$ ) at any given $\eta / \eta_{0}$.

\section{Moments of the energy dissipation rate}

Dissipation moments $\left\langle\varepsilon^{n}\right\rangle /\langle\varepsilon\rangle^{n}$ reflect variations in the smallest dissipation scales as a function of $y^{+}$and $R e_{\tau}$, particularly for high $n$. Figure 4 shows that the $n=2-4$ 


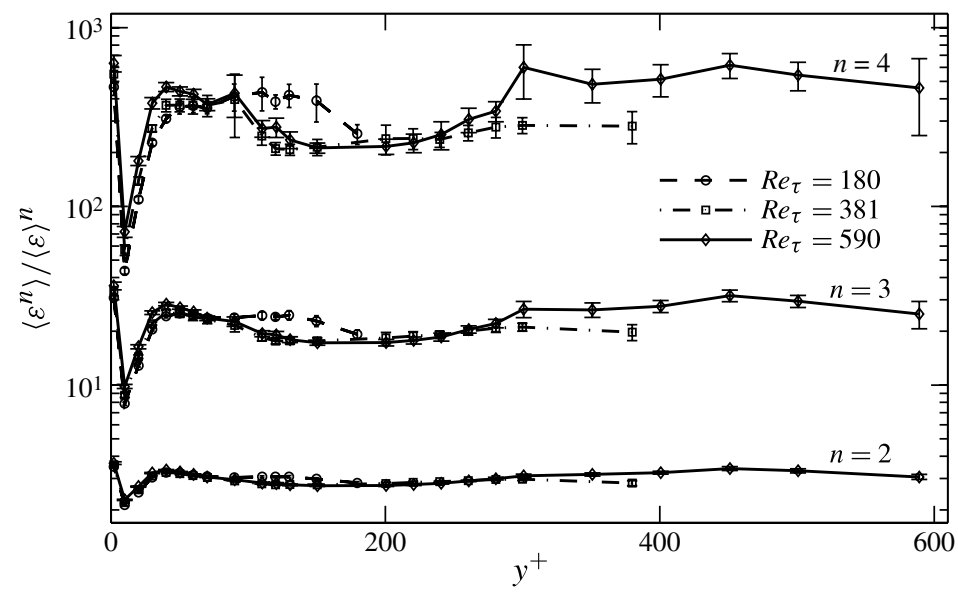

FIGURE 4. Variation of local dissipation moments $\left\langle\varepsilon^{n}\right\rangle /\langle\varepsilon\rangle^{n}$ with $y^{+}$for $n=2-4$. Error bars show $90 \%$ confidence limits.

moments are similar for all values of $R e_{\tau}$ for $y^{+} \lesssim 100$, but above this location, the $R e_{\tau}=180$ moments are larger than those for the higher values of $R e_{\tau}$. This difference may be explained by the bursting (Adrian 2007) of coherent structures away from the wall towards the channel centreline. For small $R_{\tau}$, this would introduce high $\varepsilon$ amplitudes into the bulk flow region and give the increased $\left\langle\varepsilon^{n}\right\rangle /\langle\varepsilon\rangle^{n}$ observed for $R e_{\tau}=180$ in figure 4. The moments for $R e_{\tau}=381$ and 590, by contrast, remain similar up to $y^{+} \approx 200$, and are relatively constant in both cases from $y / L \approx 0.5$ to the centreline.

Estimates of the $90 \%$ confidence intervals in figure 4 indicate that there is relatively large statistical variability in the $n=4$ moments, particularly for $R e_{\tau}=590$. These intervals have been estimated using a moving-block bootstrap method (Garcia, Jackson $\&$ Garcia 2005) on the time-varying values of $\left\langle\varepsilon^{n}\right\rangle_{x z, s}$, i.e. the normalized moments are calculated separately in each snapshot over $x-z$ planes and symmetric (s) halves of the channel. The block sizes used in the bootstrap analysis are equal to one convective time unit, $t_{c}$, for each $R e_{\tau}$. This corresponds to blocks consisting of 10,5 and 5 snapshots for $R e_{\tau}=180,381$ and 590, respectively. Obtaining greater convergence for the high-order moments shown in figure 4 requires even longer time series than those used here, which is currently beyond the limit of our available computational resources.

At the centreline, the turbulence is found to be essentially homogeneous and isotropic, and the resulting moments can be compared with prior results for homogeneous isotropic turbulence (Schumacher et al. 2007; Donzis et al. 2008). Figure 5 shows that the moments for the two higher values of $R e_{\tau}$ follow similar scaling with large-scale Reynolds number $R e$ (table 1). In particular, the $n=2-4$ moments for $R e_{\tau}=381$ and 590 are in good agreement with the scaling

$$
\left\langle\varepsilon^{n}\right\rangle \sim R e^{d_{n}},
$$

where $d_{n}=n+\zeta_{4 n} /\left(\zeta_{4 n}-\zeta_{4 n+1}-1\right)$, as shown by Yakhot (2006). Calculation of these exponents for homogeneous isotropic turbulence was shown to give $d_{2}=0.152$, $d_{3}=0.476$, and $d_{4}=0.978$ (Schumacher et al. 2007). Figure 5 shows, however, that the moments for the $R e_{\tau}=180$ case are systematically greater than the scaling laws 


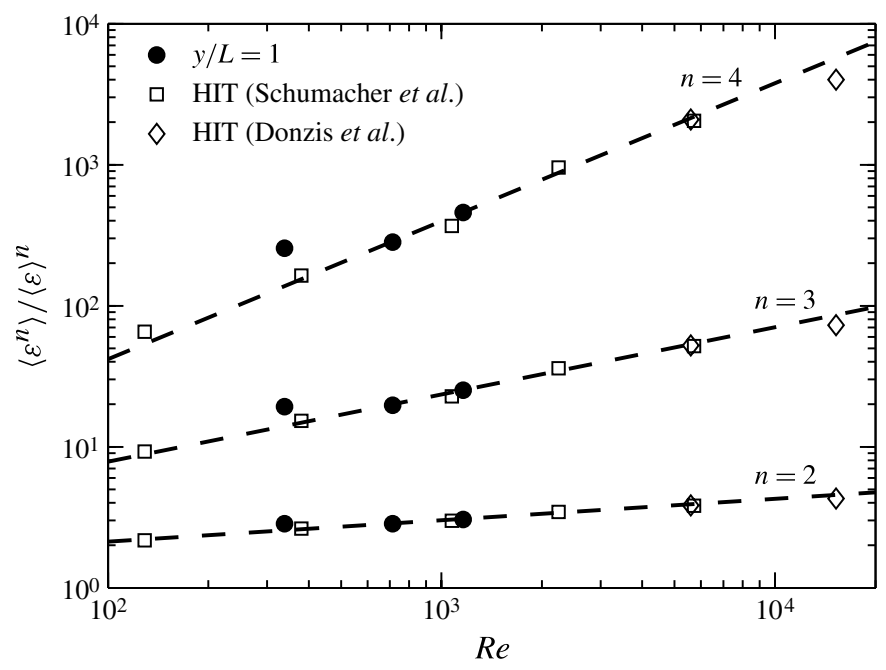

FIGURE 5. Scaling of dissipation moments $\left\langle\varepsilon^{n}\right\rangle /\langle\varepsilon\rangle^{n}$ with $R e$ from table 1 for $n=2-4$. Centerline $(y / L=1)$ moments are compared with DNS of homogeneous isotropic turbulence (HIT) from Schumacher et al. (2007) and Donzis et al. (2008). DNS results of HIT have been rescaled by constant factors at each $n$ to allow comparisons with the present results.

and all other data. This may again be due to the bursting of coherent structures away from the walls, which dominate the entire channel for small $\operatorname{Re}_{\tau}$ (see Boeck et al. 2010).

\section{Summary and conclusions}

Our analysis shows that p.d.f.s of the local dissipation scale and the Reynoldsnumber dependence of the high-order dissipation moments agree closely with results in homogeneous isotropic turbulence for the bulk of the channel and for sufficiently large $R e_{\tau}$. The p.d.f.s $Q\left(\eta / \eta_{0}\right)$ are also consistent with those from prior turbulent pipe-flow experiments by Bailey et al. (2009). These results suggest that there is a universal (flow-independent) behaviour of the scales, $\eta$, at which inertial and viscous processes are in approximate balance. This universality persists for much of the channel (particularly for the highest $R e_{\tau}$ examined here) and is only lost in the near-wall region. The p.d.f.s $Q\left(\eta / \eta_{0}\right)$ remain essentially unchanged for $y^{+} \gtrsim 100$ (even when using velocity increments in spanwise and streamwise directions), but systematic variations are observed for all $\operatorname{Re}_{\tau}$ as the wall is approached. Similar variations were also observed in the moments of $\varepsilon$, most likely due to the presence of coherent vortical structures near the walls (Adrian 2007). For small $R e_{\tau}$, these structures dominate the entire channel, resulting in departures of the dissipation moments from the predicted $R e$-scaling observed at $R e_{\tau}=180$ (see figure 5). In order to further examine the universality of $\eta$ and $\varepsilon$, in the future we would like to explore the robustness of these results for further shear flows, e.g. flows affected by Lorentz forces.

\section{Acknowledgements}

We thank the DEISA Consortium (www.deisa.eu), co-funded by the EU FP6 project RI-031513 and the FP7 project RI-222919, for support within the DEISA 


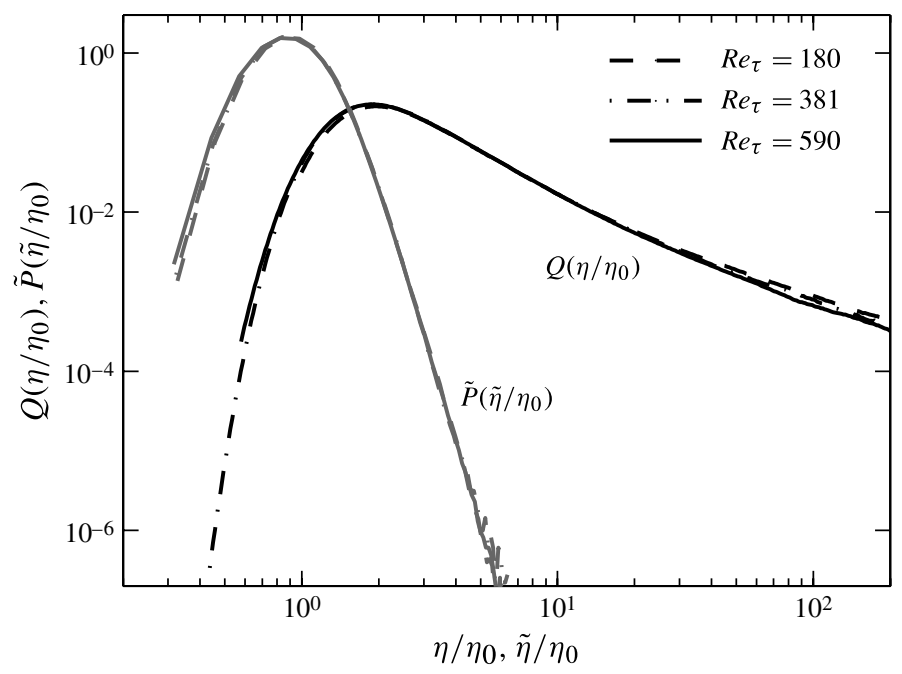

FIgURE 6. Comparisons of $Q\left(\eta / \eta_{0}\right)$ and $\tilde{P}\left(\tilde{\eta} / \eta_{0}\right)$ at the centreline for $R e_{\tau}=180,381$ and 590 .

Extreme Computing Initiative. P.E.H. was supported by a National Research Council Research Associateship Award at the Naval Research Laboratory, D.K. and T.B. by the Deutsche Forschungsgemeinschaft (Grant no 1668/5). J.S. acknowledges support by the German-Israeli Foundation under Grant 1072-6.14/2009. We thank D. A. Donzis and S. C. C. Bailey for providing data.

\section{Appendix}

This appendix explains the relation between the scales $\eta$ and $\tilde{\eta}$. The length scale, $\tilde{\eta}$, can be constructed from the local, instantaneous value of $\varepsilon$ as $\tilde{\eta}(\boldsymbol{x}, t)=\left[v^{3} / \varepsilon(\boldsymbol{x}, t)\right]^{1 / 4}$ (Sreenivasan 2004; Schumacher, Sreenivasan \& Yeung 2005). This scale is based on an analogy with the classical Kolmogorov scale $\eta_{K}$, and is distinct from the dissipation scale, $\eta$, examined here. The scale $\eta$ is obtained by requiring that the Reynolds number associated with eddies of size $\eta$ be $O(1)$. This ensures that the resulting eddies are quasi-laminar (Yakhot \& Sreenivasan 2005), and that they are thus associated with the energy-dissipating scales of motion where viscous effects become important. The Kolmogorov-type scale $\tilde{\eta}$ is, by contrast, based on dimensional arguments, and is not necessarily connected to the wide range of quasi-laminar eddy sizes at which dissipation occurs. As a result of this distinction, p.d.f.s of $\tilde{\eta} / \eta_{0}$, denoted $\tilde{P}\left(\tilde{\eta} / \eta_{0}\right)$, are considerably different from the corresponding p.d.f.s of $\eta / \eta_{0}$, as shown at the centreline in figure 6 . In particular, $\tilde{P}\left(\tilde{\eta} / \eta_{0}\right)$ has a maximum at $\tilde{\eta} / \eta_{0} \approx 1$, and a substantially less broad right tail than $Q\left(\eta / \eta_{0}\right)$. Similar differences between the two p.d.f.s have been observed in prior studies of homogeneous isotropic turbulence (e.g. in Schumacher et al. 2005; Schumacher 2007). It should be noted, however, that - consistent with the behaviour of $Q\left(\eta / \eta_{0}\right)$ - there are only weak variations in the p.d.f.s of $\tilde{\eta} / \eta_{0}$ with $\operatorname{Re}_{\tau}$ for the present data.

The differences between $\tilde{P}(\tilde{\eta})$ and $Q(\eta)$ can be examined analytically by considering homogeneous isotropic turbulence (which, as shown in the present study, is approximately the type of turbulence found at the centreline for large $\operatorname{Re}_{\tau}$ ) and by 
noting that $\varepsilon$ in such situations is given by

$$
\varepsilon=15 v\left(\partial_{x} u_{x}^{\prime}\right)^{2} \approx 15 v u_{\eta}^{2} / \eta^{2},
$$

where $\eta$ is small and we have introduced $u_{\eta} \equiv\left(\delta_{\eta} u_{x}^{\prime}\right)$ to simplify the notation. Equation (A 1) is approximately valid for a range of small $\eta$ and becomes exact as $\eta \rightarrow 0$. From (A 1) and the definition of $\tilde{\eta}$, for a given small $\eta$ we can write

$$
\tilde{\eta} \approx \frac{\eta}{\left(15 R e_{\eta}^{2}\right)^{1 / 4}}
$$

where $\operatorname{Re}_{\eta} \equiv u_{\eta} \eta / \nu$. The p.d.f. of $\tilde{\eta}$ is then given by

$$
\tilde{P}(\tilde{\eta}) \approx\left|\frac{\mathrm{d} R e_{\eta}}{\mathrm{d} \tilde{\eta}}\right| P_{\operatorname{Re}_{\eta}}\left(\frac{\eta^{2}}{15^{1 / 2} \tilde{\eta}^{2}}\right)=\frac{2 \eta^{2}}{15^{1 / 2} \tilde{\eta}^{3}} P_{\operatorname{Re}_{r}}\left(\frac{\eta^{2}}{15^{1 / 2} \tilde{\eta}^{2}} \mid r=\eta\right),
$$

where $P_{R e_{\eta}}$ denotes the p.d.f. of all Reynolds numbers in the flow over the scale $\eta$. We have defined $P_{\operatorname{Re}_{\eta}}(c) \equiv P_{R e_{r}}(c \mid r=\eta)$, where $r$ is an arbitrary scale and $c$ is some value of $R_{r}$. This conditional p.d.f. is formed from the set of all Reynolds numbers, $\operatorname{Re}_{r}$, over the scale $r=\eta$ in the flow. Equation (A 3) can be related to $Q(\eta)=P_{r}\left(\eta \mid R e_{r}=c\right)$ by noting that (using Bayes' theorem)

$$
Q(\eta)=A(\eta, c) P_{R_{r}}(c \mid r=\eta),
$$

where $A(\eta, c) \equiv P_{r}(\eta) / P_{R e_{r}}(c)$, and $P_{r}$ and $P_{R e_{r}}$ are unconditional p.d.f.s over the entire flow. Comparison of (A 3 ) and (A 4) reveals several differences between $\tilde{P}(\tilde{\eta})$ and $Q(\eta)$. Most notably, for a given $\eta$, each value of $\tilde{\eta}$ in (A 3) corresponds to a different value of $R e_{\eta}$, which is not necessarily $O(1)$. The p.d.f. $\tilde{P}(\tilde{\eta})$ thus includes contributions from regions of size $\eta$ over which inertial and viscous forces are not in approximate balance. By contrast, $Q(\eta)$ in (A 4) is restricted to $R e_{\eta}=c$ for all $\eta$, where $c \approx 1$ is used in the present study to isolate quasi-laminar regions in the flow. It should also be noted that (A 1) and (A 3) are only accurate for small $\eta$, and thus $\tilde{P}(\tilde{\eta})$ is only connected here to $Q(\eta)$ at small scales.

\section{REFERENCES}

Abe, H. \& Antonia, R. A. 2011 Scaling of normalized mean energy and scalar dissipation rates in a turbulent channel flow. Phys. Fluids 23, 055104.

Adrian, R. J. 2007 Hairpin vortex organization in wall turbulence. Phys. Fluids 19, 041301.

Bailey, S. C. C., Hultmark, M., Schumacher, J., Yakhot, V. \& Smits, A. J. 2009 Measurement of dissipation scales in turbulent pipe flow. Phys. Rev. Lett. 103, 014502.

Biferale, L. 2008 A note on the fluctuation of dissipative scale in turbulence. Phys. Fluids 20, 031703.

Boeck, T., Krasnov, D. \& Schumacher, J. 2010 Statistics of velocity gradients in wall-bounded shear flow turbulence. Physica D 239, 1258-1263.

Donzis, D. A., Yeung, P. K. \& SReenivasan, K. R. 2008 Dissipation and enstrophy in isotropic turbulence: resolution effects and scaling in direct numerical simulations. Phys. Fluids 20, 045108.

GARCIA, C. M., JACKSON, P. R. \& GARCiA, M. H. 2005 Confidence intervals in the determination of turbulence parameters. Exp. Fluids 40, 514-522.

Hamlington, P. E., Krasnov, D., Boeck, T. \& Schumacher, J. 2012 Statistics of energy dissipation rate and local enstrophy in turbulent channel flow. Physica D 241, 169-177.

Hoyas, S. \& JimeneZ, J. 2006 Scaling of the velocity fluctuations in turbulent channels up to $\operatorname{Re}_{\tau}=2003$. Phys. Fluids 18, 011702. 
Ishinara, T., Gotoh, T. \& Kaneda, Y. 2009 Study of high-Reynolds number isotropic turbulence by direct numerical simulation. Annu. Rev. Fluid Mech. 41, 165-180.

Kim, J., Moin, P. \& Moser, R. D. 1987 Turbulence statistics in fully developed channel flow at low Reynolds number. J. Fluid Mech. 177, 133-166.

Kolmogorov, A. N. 1941 The local structure of turbulence in incompressible fluid for very large Reynolds number. Dokl. Akad. Nauk SSSR 30, 299-303.

Moser, R. D., Kim, J. \& MAnsour, N. N. 1999 Direct numerical simulation of turbulent channel flow up to $R e_{\tau}=590$. Phys. Fluids 11, 943-945.

NelKin, M. 1990 Multifractal scaling of velocity derivatives in turbulence. Phys. Rev. A 42, 7226-7229.

Paladin, G. \& Vulpiani, A. 1987 Degrees of freedom of turbulence. Phys. Rev. A 35, 1971-1973.

SCHUMACHER, J. 2007 Sub-Kolmogorov scale fluctuations in fluid turbulence. Europhys. Lett. 80, 54001.

Schumacher, J., Sreenivasan, K. R. \& Yakhot, V. 2007 Asymptotic exponents from low-Reynolds number flows. New J. Phys. 9, 89.

Schumacher, J., SReenivasan, K. R. \& Yeung, P. K. 2005 Very fine structures in scalar mixing. J. Fluid Mech. 531, 113-122.

SHE, Z. S. \& LÉvÊQUe, E. 1994 Universal scaling laws in fully developed turbulence. Phys. Rev. Lett. 72, 336-339.

SReEnivasAn, K. R. 2004 Possible effects of small-scale intermittency in turbulent reacting flows. Flow Turbul. Combust. 72, 115-131.

Sreenivasan, K. R. \& Antonia, R. A. 1997 The phenomenology of small-scale turbulence. Annu. Rev. Fluid Mech. 29, 435-472.

Wallace, J. M. \& Vukoslavcević, P. V. 2010 Measurement of the velocity gradient tensor in turbulent flows. Annu. Rev. Fluid Mech. 42, 157-181.

YАкнот, V. 2006 Probability densities in strong turbulence. Physica D 215, 166-174.

YAKHOt, V. \& SREEnivasAn, K. R. 2005 Anomalous scaling of structure functions and dynamic constraints on turbulence simulations. J. Stat. Phys. 121 (5), 823-841.

ZHOU, Q. \& XIA, K.-Q. 2010 Universality of local dissipation scales in buoyancy-driven turbulence. Phys. Rev. Lett. 104, 124301. 